\title{
Successful treatment of severe burn patients with multiple organ dysfunction syndrome: A case report
}

\author{
Lingfeng Wang ,'Yongdong Li, Xiyuan Xu, Ji Chen, Weiqing Wang, Zaiqing Huang, Lihua Zhang \\ Inner Mongolia Institute of Burns, The Third Affiliated Hospital of Inner Mongolia Medical University, Hohhot, China
}

Received: July 10, 2012

Accepted: August 1, 2012

Online Published: October 15, 2014

DOI: $10.14725 /$ dcc.v1n1p1

URL: http://dx.doi.org/10.14725/dcc.v1n1p1

\begin{abstract}
Multiple organ dysfunction syndrome is the presence of altered organ function of two or more organ systems in acute ill patients with severe trauma, burn, shock and infection. In this case, the patient with burn area amounted to $95 \%$ and the third-degree burn was up to $90 \%$. He underwent gastrointestinal tract, blood clotting, lung, brain, heart, liver dysfunction, and cardiac arrest for 30 minutes during the courses of treatment, and was discharged from the hospital after 108 days on the basis of comprehensive treatment and repeated skin grafting.
\end{abstract}

Key Words: Multiple organ dysfunction syndrome, Burns, Clinical

\section{Medical record}

\subsection{General information}

A 36-year-old male patient with fire-breathing injury due to blast furnace was referred to local hospital for rehydration therapy. He was admitted to our hospital after a longdistance conveying 7 hours after the injury. The patient was conscious, T $36.7^{\circ} \mathrm{C}, \mathrm{P} 110 / \mathrm{min}, \mathrm{R} 22 / \mathrm{min}$, blood pressure was not measured due to limb swelling. Besides that, he was presented with mild irritability, sense of thirsty, cold limbs and poor peripheral circulation. About $5 \%$ of normal skin was preserved on head, periumbilical region, waist and back, perineum and double foot, leaving the rest part to be burn wound. The burn wounds at face and neck, trunk, limbs were diagnosed to be leather like change, subcutaneous venous embolism net could be visualized and tenderness disappeared. $5 \%$ of burn wound were seen putrid skins exfoliation of basal red and at neck and feet without sensitivity at neck and feet.

\section{Laboratory examination}

WBC $29.7 \times 10^{9} / \mathrm{L}$, RBC $5.63 \times 10^{12} / \mathrm{L}, \mathrm{Hb} 178 \mathrm{~g} / \mathrm{L}$, PLT $186 \times 10^{9} / \mathrm{L}$, HCT $39.9 \%$. Glu $5.1 \mathrm{mmol} / \mathrm{L}$, blood urea nitrogen $6.58 \mathrm{mmol} / \mathrm{L}$, serum creatinine $74.26 \mu \mathrm{mol} / \mathrm{L} . \mathrm{K}^{+}$ $3.62 \mathrm{mmol} / \mathrm{L}, \mathrm{Na}^{+} 139.2 \mathrm{mmol} / \mathrm{L}, \mathrm{Cl}^{-} 101.2 \mathrm{mmol} / \mathrm{L}$. Blood gas analysis: $\mathrm{pH} 7.292, \mathrm{PO}_{2}: 75.3 \mathrm{mmHg}, \mathrm{PCO}_{2}$ : $31.9 \mathrm{mmHg}, \mathrm{BE}-10.0 \mathrm{mmol} / \mathrm{L}, \mathrm{BB} 37.9 \mathrm{mmol} / \mathrm{L}, \mathrm{HCO}_{3}^{-}$ $14.9 \mathrm{mmol} / \mathrm{L}, \mathrm{SpO}_{2} 92.3 \%$.

\section{Primary diagnosis}

95\% of systemic flame burns, $90 \%$ of third-degree burns, $5 \%$ of deep second-degree burns.

\footnotetext{
*Correspondence: Lingfeng Wang, E-mail: nmgbgyyyy@163.com. Address: Inner Mongolia Institute of Burns, The Third Affiliated Hospital of Inner Mongolia Medical University, Hohhot, China 


\section{Diagnosis and treatment}

The patient received anti-shock treatment, peripherally inserted central catheter (PICC), rapid fluid replacement, debridement, limbs relief incision and silver sulfadiazine paste for burn wound after admission. Intravenous drip of cefoperazone sodium and sulbactam sodium for injection $4.0 \mathrm{~g}$ was used once every 12 hours for anti-infection. Besides that, an additional administration of compound salvia miltiorrhiza injection, Omeprazole sodium for injection, Adenosine disodiu, Glutamine granules, Bifid triple viable capsules, Fructose 1,6-diphosphate, vitamin E, vitamin Bco, vitamin A and other drug treatment was employed as well. At night, he vomited $200 \mathrm{ml}$ coffee ground vomitus, fecal occult blood testing was positive, four items of blood coagulation included: PT $56.3 \mathrm{~s}$ (control group $13.1 \mathrm{~s}$ ), APTT $80.9 \mathrm{~s}$ (control group $34.3 \mathrm{~s}$ ), TT $14.4 \mathrm{~s}$ (control group $13.9 \mathrm{~s}$ ), fibrinogen $4.45 \mathrm{~g} / \mathrm{L}$, was significantly longer than usual. Norepinephrine iced saline nasal feeding and intravenous drip of tranexamic acid treatment was given once the diagnosis of stress ulcer hemorrhage of digestive tract was confirmed.

The total amount of fluid infusion for the first 24 hours was $10,400 \mathrm{ml}$, including plasma $2,900 \mathrm{ml}$, hetastarch $1,000 \mathrm{ml}$, $5 \%$ sodium bicarbonate saline $650 \mathrm{ml}$, physiological saline $3,850 \mathrm{ml}$ and $2,000 \mathrm{ml}$ of $5 \%$ glucose, and urine output within 24 hours was $2,115 \mathrm{ml}$, an average of $88 \mathrm{ml} / \mathrm{h}$. The patient felt dyspnea on the second day of admission. The laboratory examination showed: WBC $30.1 \times 10^{9} / \mathrm{L}$, RBC $7.46 \times 10^{12} / \mathrm{L}$, Hb $227 \mathrm{~g} / \mathrm{L}$, PLT $85 \times 10^{9} / \mathrm{L}$, HCT $65.1 \%$, $\mathrm{K}^{+} 3.95 \mathrm{mmol} / \mathrm{L}, \mathrm{Na}^{+} 150.6 \mathrm{mmol} / \mathrm{L}, \mathrm{Cl}^{-} 109.7 \mathrm{mmol} / \mathrm{L}$. Blood gas analysis of blood oxygen saturation decreased highly suggestive of metabolic acidosis so that thoracic fasciotomy was operated at once. Postoperative blood gas examination showed: $\mathrm{PO}_{2} 51 \mathrm{mmHg}, \mathrm{PCO}_{2} 24 \mathrm{mmHg}$, indicating that hypoxia was not released, therefore, immediate tracheotomy and ventilator assisted breathing (SMIV + PEEP) were carried out, then dyspnea eased slightly. The laboratory test result on the third day after injury included: WBC $10.2 \times 10^{9} / \mathrm{L}$, RBC $7.46 \times 10^{12} / \mathrm{L}, \mathrm{Hb} 173 \mathrm{~g} / \mathrm{L}$, PLT $98 \times 10^{9} / \mathrm{L}, \mathrm{K}^{+} 4.42 \mathrm{mmol} / \mathrm{L}, \mathrm{Na}^{+} 150.8 \mathrm{mmol} / \mathrm{L}, \mathrm{Cl}^{-}$ $111.1 \mathrm{mmol} / \mathrm{L}$. Four items of blood coagulation: PT $42.3 \mathrm{~s}$ (control $13.3 \mathrm{~s}$ ), APTT $43.1 \mathrm{~s}$ (control $34.3 \mathrm{~s}$ ), TT $32.6 \mathrm{~s}$ (control $13.9 \mathrm{~s}$ ), fibrinogen: $4.81 \mathrm{~g} / \mathrm{L}$. The patient's temperature, heart rate was normal, breathed slightly faster. $50 \%$ limbs wound excision surgery was performed under general anesthesia, including particulate skin autograft, pulp of dermis transplantation, allogeneic skin cover and harvesting of skin grafts at head and trunk. Postoperative treatment of cefoperazone sodium $4.0 \mathrm{~g}$ by intravenous drip, bid, oral administration of sporanox $20 \mathrm{mg}$, qd, fleroxacin $0.2 \mathrm{~g}$ by intravenous drip, bid, was given, and blood and nutrition support were strengthened. The symptom of systematic swelling was significantly relieved on the fourth day after injury. Laboratory examination showed: WBC $4.9 \times 10^{9} / \mathrm{L}, \mathrm{RBC} 4.56 \times 10^{12} / \mathrm{L}, \mathrm{Hb} 140 \mathrm{~g} / \mathrm{L}$,
PLT $51 \times 10^{9} / \mathrm{L}, \mathrm{HCT} 42.7 \%$. $\mathrm{K}^{+} 3.81 \mathrm{mmol} / \mathrm{L}, \mathrm{Na}^{+}$ $143.7 \mathrm{mmol} / \mathrm{L}, \mathrm{Cl}^{-} 105.2 \mathrm{mmol} / \mathrm{L}$, Blood gas analysis: $\mathrm{pH}$ 7.484, $\mathrm{PO}_{2} 46.9 \mathrm{mmHg}, \mathrm{PCO}_{2} 22.7$, BE $-3.3 \mathrm{mmol} / \mathrm{L}, \mathrm{BB}$ $44.6 \mathrm{mmol} / \mathrm{L}, \mathrm{HCO}_{3}^{-} 16.5 \mathrm{mmol} / \mathrm{L}, \mathrm{SpO}_{2} 85.7 \%$. Body temperature up to $38^{\circ} \mathrm{C}-39.8^{\circ} \mathrm{C}$, heart rate 130 beats/min. $25 \%$ limbs wound excision surgery was performed under general anesthesia on the sixth day, including particulate skin autograft, pulp of dermis transplantation, allogeneic skin cover and harvesting of skin grafts at head and trunk. Amikacinisulfatis $0.2 \mathrm{~g}$ by intravenous drip, bid, was added. According to the results of bacterial culture, wound dressing intravenous nutritional support therapy was enhanced, oral administration of Fresubin was remained according to the results of bacterial culture. His temperature was up to $40^{\circ} \mathrm{C}$, heart rate $140-150$ beats $/ \mathrm{min}$, with occasional delirium on the ninth day after injury, scab formed at double lower limbs residual wound and interface of surgery area with more secretion. 5\% limbs wound excision surgery was performed under general anesthesia, including particulate skin autograft, pulp of dermis transplantation, allogeneic skin cover and harvesting of skin grafts at rear quarters to control wound infection.

The patient suffered from ventricular fibrillation, cardiac arrest during the surgery and recovered after chest compressions for 30 minutes. He was presented with irritability, delirium, sleepiness on the next day, and was treated with tienam $1.0 \mathrm{~g}$ by intravenous drip, tid, and vancomycin hydrochloride was $0.8 \mathrm{~g}$ by intravenous drip, bid, due to systematic infection, and burn wound treatment were intensified. $4 \%$ limbs wound excision surgery was performed under general anesthesia, including particulate skin autograft, allogeneic skin cover and harvesting of skin grafts at head and trunk. Laboratory examination showed: WBC $13.7 \times 10^{9} / \mathrm{L}$, RBC $2.17 \times 10^{12} / \mathrm{L}, \mathrm{Hb} 69 \mathrm{~g} / \mathrm{L}$, PLT $242 \times 10^{9} / \mathrm{L}$ with temperature $38.9^{\circ} \mathrm{C}$ on the 21 st day after injury. As a consequence, the application of tienam and vancomycin hydrochloride was changed into piperacillin followed by blood transfusion. The assistance of a ventilator was suspended for conventional oxygen inhalation on the next day and the patient had no dyspnea. Blood gas analysis result included: $\mathrm{pH} 7.435, \mathrm{PO}_{2} 50.4 \mathrm{mmHg}, \mathrm{PCO}_{2}$ $29.3 \mathrm{mmHg}, \mathrm{BE}-2.6 \mathrm{mmol} / \mathrm{L}$, BB $45.3 \mathrm{mmol} / \mathrm{L}, \mathrm{HCO}_{3}^{-}$ $19.1 \mathrm{mmol} / \mathrm{L}, \mathrm{SpO}_{2} 86.4 \%$. Most of the burn wounds were healed with about $7 \%$ residual wounds after 70 days. The patient's body temperature, heart rate, respiration were normal, and underwent residual wound skin grafting operation 3 times and was discharged from hospital after 108 days. Burn wound and phlegm cultivated pseudomonas aeruginosa, baumanii, staphylococcus aureus, methicillin-resistant staphylococcus epidermidis, yeast-like fungi and blood culture sterile growth. During the treatment, serum alanine aminotransferase was as high as $160 \mathrm{U} / \mathrm{L}$, aspartate aminotransferase as high as $154 \mathrm{U} / \mathrm{L}$, total bilirubin was up to $22.4 \mu \mathrm{mol} / \mathrm{L}$ so that glucuronolactone and inosine treatment was given. Besides that, the patient also received traditional 
Chinese medicine treatment during hospitalization.

\section{Discussion}

\subsection{Dr. Yong-dong Li}

Dr. Yong-dong Li is the chief physician and associate professor of the department of cardiology at The Third Affiliated Hospital of Inner Mongolia Medical University, specializing in the treatment and research of cardiovascular disease intervention.

The patient is a young man, who had no history of hypertension and diabetes, no family history of heart disease, no chest tightness, nor chest pain history. The result of physical examination included: The patient could lie on his back, double lung breath sounds resonance, heart boundary was normal, heart rate $110 / \mathrm{min}$, showing regularity in the force of heartbeat. Noise was not heard in each valve by auscultation, and heart failure was not found. Results of ECG examination indicated sinus rhythm and normal ECG. No severe arrhythmia, no atrioventricular block nor sinus arrest was detected by ECG monitoring before the surgery. Laboratory examination demonstrated that troponin as specific marker of myocardial injury was at normal level so that there was no basis for cardiac arrest caused by organic heart disease. Preoperative examination showed $\mathrm{K}^{+} 3.81 \mathrm{mmol} / \mathrm{L}$ without hyperkalemia performance, blood gas analysis showed mild respiratory alkalosis without hypercapnia so that cardiac arrest caused by hyperkalemia and severe acidosis was excluded.

The following factors which are related to a large area burns with shock and cardiac arrest during intraoperative period include: (1) hypovolemia caused by a lot of fluid loss. (2) vagus nerve hyperexcitability induced by tissue stretch and posture changes of the organization during the surgery. (3) hypoxia. The heart was at low perfusion and hypoxia state before cardiac arrest as the preoperative blood gas analysis showed hypoxemia and hypovolemic before the surgery.

Efforts at resuscitation lasting more than 30 minutes appear to be uniformly successful when the patient was presented with cardiac arrest. The main factors which contributed to the success of the surgery were: (1) Timely detection of cardiac arrest (ventricular fibrillation of the patient) provides the possibility of successful surgery. Effective chest compressions were performed ensure that the blood supply to the coronary arteries and the brain when cardiac arrest was detected, which proved to be crucial to the surgery. There is a casual relationship between cardiopulmonary resuscitation start time and prognosis. ${ }^{[1]}$ (2) Recovery adrenergic drugs is another the most important factor which could not be ignored. But there are large individual differences in the amount. (3) An effective complement of blood volume. (4) Cardiopulmonary resuscitation (CPR) training for the anesthesiologist and operation room nurses, together with team-work spirit, is very important for improving the success rate of recovery.

An effective CPR adheres to the latest guide "2010 American Heart Association (AHA) cardiopulmonary resuscitation and emergency cardiovascular care (ECC) guidelines". Compared with 2005 version, the latest guideline made great changes. (1) CPR process transforms from A-B-C (development of the airway, artificial ventilation, chest compressions) for the C-A-B. Because cardiac arrest occurs at all ages so that most of fibrillation and pulseless ventricular tachycardia, artificial respiration in the course of implementation chest compressions are often delayed. ${ }^{[2]}$ (2) effective chest compressions to minimize disruption. Chest compressions required 100 beats/min in 2005, and compressions at present are more than 100 beats/min with deeper depth of at least $5 \mathrm{~cm}$ for adults (pressing ventilation ratio is still 30:2). Any unnecessary interruption of chest compressions can reduce the effectiveness of CPR. (3) The significance of teamwork during cardiopulmonary resuscitation operation. ${ }^{[3]}$

Cardiac arrest is a catastrophic event during anesthesia which poses a serious threat to the lives of patients. Smooth anesthesia combined with good care makes it possible for the prevention and early detection of cardiac arrest. Invasive arterial pressure monitoring during cardiac electrical interference is of great assist to detect cardiac arrest. Central venous access is the most efficient and effective route of administration for the treatment of cardiac arrest. While continuous cardiac massage is the key to a successful recovery. Gentle surgical procedures and intraoperative monitoring are also important to reduce and prevent surgery cardiac arrest.

\subsection{Dr. Xi-yuan Xu}

Dr. Xi-yuan Xu is the chief physician and associate professor of respiratory and critical care medicine section at The Third Affiliated Hospital of Inner Mongolia Medical University, specializing in respiratory and critical care medicine.

Acute lung injury (ALI)/Acute respiratory distress syndrome (ARDS) induced by inhalation injury, shock, infection and other factors is more likely to occur after severe burns. ${ }^{[4]}$ ARDS is a kind of acute respiratory failure with high mortality, mainly presented with acute respiratory distress and hypoxemia. The main risk factors are the greater total area and third-degree burns complicated with severe inhalation injury. Recovery or delayed recovery during hospital treatment turned out to be non-effective. Untreated wound has a tendency to cause serious systemic infection, sepsis, and so on. There is a potential risk factor of ARDS, such as associated with inhalation injury, shock, or severe systemic infection during extensive burns treatment process. The patient in the case was with severe burns, shock and 
infection, was prone to ARDS so that early detection and timely prevention were required.

The key to the treatment of ARDS is to keep the respiratory tract patency. ${ }^{[5]}$ An effective reduction technique to reduce the eschar compression on vascular and trachea is suggested during the formation of edema or eschar as the burn wounds were at neck and chest. Moreover, improved the scope of activities of thorax and diaphragm, increased the effective ventilation at the same time. The patient underwent thoracic fasciotomy in due time, which was conducive to the improvement of ARDS. Early tracheotomy not only prevents the respiratory tract obstruction but also can timely remove sputum and necrosis of the mucosa for burn patients with inhalation injury. This patient should be treated with early tracheotomy to prevent difficulty in breathing induced by respiratory tract obstruction causing, and thus aggravated the condition. Mechanical ventilation should be performed once tracheotomy was conducted, guided by lung protective ventilation strategy. ${ }^{[6]}$ That is, low tidal volume, low positive end-expiratory pressure (PEEP) and permissive hypercapnia. Tidal volume was set to $6-8 \mathrm{ml} / \mathrm{kg}$, PEEP 3-10 $\mathrm{cmH}_{2} \mathrm{O}$, assisted respiration frequency setted at $12-20 / \mathrm{min}$, fraction of inspired oxygen $\left(\mathrm{FiO}_{2}\right)$ maintained at $40 \%-60 \%$. The application of mechanical ventilation strategy was on the basis of conditions of patients and blood gas analysis results. The early airway humidification and lavation together with early fiberoptic bronchoscope examination and treatment. Airway management and bronchoscopy examination should be enhanced for patients under mechanical ventilation.

Some studies also demonstrated inflammatory cells aggregation and deposition, and adhesion in patients with lung injury so that release of cytokines and mediators of inflammation caused extensive damage of vascular endothelial cells and other tissue cells in burn patients. ${ }^{[7]}$ Continuous renal replacement therapy (CRRT) can decrease the content of serum inflammatory mediators and cytokines, thereby weakening inflammation responses of the lung, reducing the lung injury and pulmonary edema, significantly improving pulmonary function. ${ }^{[8]}$ So the feasible CRRT treatment can accelerate the healing time of ARDS, reduce hospitalization time, therefore decrease hospitalization expenses.

\subsection{Dr. Ji Chen}

Dr. Ji Chen is the chief physician and professor of the department of gastroenterology at The Third Affiliated Hospital of Inner Mongolia Medical University, specializing in digestive disorders treatment and endoscopic diagnosis and treatment of diseases of the digestive system.

The patient was diagnosed as severe extensive burns, hypovolemic shock, severe systemic infections accompanied by upper gastrointestinal bleeding, liver and lung damage, coagulation disorders, and acid-base balance disorders. He had nausea at night upon hospital admission, vomited coffee ground vomitus $200 \mathrm{ml}$ and fecal occult blood testing (+), fortunately hemoglobin was normal. Regular daily dose of intravenous omeprazole was given in 21 days when burn hemoglobin dropped to $69 \mathrm{~g} / \mathrm{L}$, long-term use of large doses of immunosuppressive agents or non-steroidal antiinflammatory drug (NSAID) history was denied, and the patient had no recent history of peptic ulcer or upper gastrointestinal bleeding history. Extensive burns, shock, severe systemic infections and brain injury are the stress factors that caused acute gastric mucosal lesion (AGML) and ultimately may lead to gastrointestinal bleeding. AGML prevention and treatment principles: (1) Earlier alimentation makes it possible to neutralize stomach acid and enhance gastric mucosal barrier function if the patient's condition permits. If hypoproteinemia, electrolyte and acid-base balance disorders occurs, they should be promptly added and adjusted. (2) Stomach lavage with iced saline boosts stomach vasoconstriction, reduces gastric acid secretion, promotes hemostasis and reveals the bleeding speed from the blood containing flushing fluid. (3) Acid-suppressing agents: proton pump inhibitor omeprazole or lansoprazole can significantly increase gastric $\mathrm{pH}(\geq 6)$, which promotes platelet aggregation and prevents thrombolysis, and is to create the necessary conditions for stomach bleeding. (4) Antacids: aluminum hydroxide, aluminum magnesium carbonate and $5 \%$ sodium bicarbonate solution were injected from the inner tube to make $\mathrm{pH} \geq 4$. (5) Mucosal protective agents. It includes sucralfate tablets, prostaglandin $\mathrm{E}$ and so on. The tablets were crushed and transferred to a paste and infused through stomach tube 2-3 times a day for at least two weeks. It turns out to be an effective and cheap drug. (6) Emergency endoscopy and endoscopic hemostasis treatment should be performed immediately to confirm the diagnosis if the disease could not be controlled after drug treatment.

AGML refers to clinical symptoms characterized by pathological changes in gastric mucosal damage and upper gastrointestinal bleeding, which induced by severe burns, trauma, infection, shock and other stress. AGML accounts for $22 \%-30 \%$ of upper gastrointestinal bleeding cases. Clinically, there are many different names, such as acute hemorrhagic gastritis, acute erosive gastritis, acute drug-induced gastritis, gastroduodenal ulcer, stress gastritis, and ulcer. It is more appropriate to include acute gastric mucosal damage caused by a variety of stress factors and superficial ulcers as AGML from the perspective of pathology.

The common factors that initiate AGML are: (1) severe traumatic brain injury (also known as Cushing ulcer); (2) severe burns (also called Curling ulcer); (3) after severe trauma and complex major surgery; (4) After shock, heart, lung, brain resuscitation; (5) serious systemic infection; (6) multiple organ dysfunction (MODS); (7) severe psychological stress, such as trauma, excessive tension and so on. 
AGML pathophysiological processes is very complex and is regarded as the combined result of gastric ischemia, hypoxia, the relative increase in gastric acid secretion, neuroendocrine disorders and other factors caused by the reduction of gastric mucosal defense mechanism and gastric mucosal blood flow. The diagnosis of AGML mainly counts on stress history, lesions in the two weeks after the occurrence of the primary disease abdominal discomfort, fullness, upper gastrointestinal bleeding, perforation and other symptoms. Endoscopy examination is required to establish the diagnosis of AGML, it being erosions, ulcers in the stomach.

The treatment for AGML accompanied by massive hemorrhage is a challenge. While positive medical treatment should be referred first. The most primary and basic treatment for the prevention of AGML is to timely reduce the stress factors of burns, shock, infection and so on, together with an effective treatment of the primary disease and its concurrent disease. Besides that, protect and maintain the normal function of various organs, prevent and cure organ dysfunction. Based on the basic treatment, the goal to reduce the incidence of AGML and improve the cure rate may be achieved with a deeper exploration of trauma and critical illness, a better understanding of stress response and the development of intensive care equipment and technology. ${ }^{[9]}$

\subsection{Dr. Wei-qing Wang}

Dr. Wei-qing Wang is the director of critical care medicine and associate professor at Third Affiliated Hospital of Inner Mongolia Medical University, specializing in critical care medicine.

Diagnostic analysis: (1) On distributive shock and hypovolemic shock. Though the blood pressure in patients was undetectable, the diagnosis of distributive shock and hypovolemic shock was established as the patient was presented with mild irritability, sense of thirsty, cold limbs, poor peripheral circulation, lack of adequate perfusion of microcirculation and an increase of white blood cells. Shock is divided into hypovolemic, cardiogenic, distribution and obstructive shock according to the new classification of circulation dysfunction caused by shock instead of the method of its underlying cause. Shock caused by different reasons can be manifested in the hemodynamic changes of the same or similar. Distributive shock can be caused by abnormal vascular systolic and diastolic function. Infection is the primary cause of it. Besides that, the fact that it could cause circulating blood volume reduction is relative. Blood volume distribution of the abnormal sites may lead to a decrease in effective circulating blood volume. Hypovolemic shock is mainly characterized by a significant decrease of low intravascular volume. (2) Whether respiratory tract was damage. First, the patient was burned in a confined space. Similar cases accounted for $47 \%-62 \%$ of all respiratory burns cases and accompanied by inhalation of irritating and harmful gases. Second, there were burns at head, face and neck, vibrissa was much charred, blisters, hematoma at oral and pharyngeal mucosa or with a hoarse voice, accounting for $41 \%-69 \%$ of all respiratory tract burns. Third, the patients brought up phlegm when coughing and contained black carbon-like particles, was presented with shallow breathing or dyspnea, or accompanied by large bloody foamy sputum. Early wheeze was heard during lung auscultation, accounted for $81 \%-100 \%$ of respiratory tract burns. Fourth, it indicates deep and serious burn when coughing without phlegm or no cough reflex at protuberance. Some signs are useful indicators to aid in the diagnosis of respiratory burns, for example, hoarseness, irritable cough, high pitched rhonchi at the chest suggest that damage has affected the glottis. Moreover, dyspnea, wheeze and moist rhonchi suggest that damage has affected bronchial and lung parenchyma. The emergence of rapid breathing, expiratory dyspnea, cough, hemoptysis reveal that damage has covered small airway and lung. Patients with respiratory burns at early stage manifested as a decrease in $\mathrm{PaO}_{2}$, oxygenation index $\left(\mathrm{PaO}_{2} / \mathrm{FiO}_{2}\right)$ reduction, a significant increase in blood, alveolar and arterial oxygen difference (A-a) $\mathrm{DO}_{2}$ due to airway obstruction, alveolar ventilation / perfusion imbalance, ventilation and gas exchange dysfunctions. Some believe that the increasing of $\mathrm{DO}_{2}$ is highly correlated with severe levels of respiratory burns within the first 24 hours, which could be used to estimate the severity of respiratory burns. Oxygenation index $\left(\mathrm{PaO}_{2} / \mathrm{FiO}_{2}\right)$ and accurate calculation of the value of pulmonary shunt are much associated. It indicates respiratory dysfunction if the ratio is less than 300 , severe respiratory insufficiency if the ratio is less than 200 so that mechanical ventilation is requested. Chest X-ray showed infection visible center infiltration images or diffuse and dense infiltration image if the patient was with pulmonary infection. It demonstrated the symptoms of pulmonary edema when it displayed diffuse slide-like shadows, mesenchymal images, hilar enlargement and linear or crescent shaped images. Bronchoscopy examination is needed when necessary.

Dynamic monitoring of blood lactate levels and lactate clearance rate is an important indicator of shock degrees and tissue perfusion disorders. The essence of septic shock is hypoxia so that timely treatment of hypoxia is the key to improve its prognosis. Tissue hypoxia has occurred at early shock period while the vital signs are still in normal range. Tissue hypoperfusion and hypoxia have already existed and lactate levels have increased before any changes of blood stream dynamics monitor indicators. If the blood lactate $>4 \mathrm{mmol} / \mathrm{L}$, with hypotension, its mortality is over $8 \%$, hence immediate resuscitation $(20 \mathrm{ml} / \mathrm{kg})$ is demanded. Additional administration of vasoactive drugs is needed to maintain mean arterial pulse $\geq 65 \mathrm{mmHg}, \mathrm{CVP} \geq 8 \mathrm{cmH}_{2} \mathrm{O}$ provided that low blood pressure could not be treated. ${ }^{[10]}$

Principles of antibiotic prophylaxis are early, joint, adequate and sensitive. Rational use of antibiotics has the function to 
lower the incidence rate of invasive infection. At present, Pseudomonas aeruginosa and staphylococcus aureus are the main pathogenic bacteria on burn wound infection, one of the important pathogenic bacteria of hospital infection. The patient in this case received cefoperazone sulbactam infusion, a combination with anti-bacteria, such as vancomycin, teicoplanin or linezolid is suggested. Initiative immunization and passive immunization methods show effectiveness in prevention of pseudomonas aeruginosa infection. Active immunization for patients with large burn area is injection of the polyvalent pseudomonas aeruginosa vaccine $20 \mathrm{mg} / \mathrm{kg}$ by intramuscular or intradermal injection every seven days since his admission. Infusion of passive immune globulin should be given at the same time when active immunization is conducted. Aminophylline, anisodamine or promethazine is a feasible method to relieve bronchospasm. Pulmicort with bricanyl nebulising solution is used for airways expansion, $\mathrm{N}$-acetyl cysteine for phlegm reduction, use of steroids is applied when necessary. ${ }^{[11]}$

The maintenance of effective circulating blood volume is the key to guarantee the perfusion of important organs. Plasma is the first choice of colloidal liquid for blood supplement for patients with severe burn, and appropriate amount of dextran plasma substitutes is permissible, however, the maximum daily dose of dextran is $1,000 \mathrm{ml}$. The principle component of crystalloid solution is ringer lactate solution with $5 \%$ or $10 \%$ glucose solution as basic water, yet over dose of saline infusion is the origin of hyperchloremic acidosis. Mean arterial pressure (diastolic pressures $+1 / 3$ pulse pressure) and urine volume is considered as the most reliable indicators to determine whether the amount of liquid in burn patients is sufficient. Adequate cerebral perfusion should be assured when MAP level is above $60 \mathrm{mmHg}$. Hypotension or uric quantity reduction should not be treated by high molecular colloid alone and injection speed of fluid resuscitation was valued as colloidal solution would aggravate the capillary leakage and edema. Besides that, ignore that application of albumin for patients with burn wound at early stage in that it could leak into the interstitial space and then results in very serious edema.

The use of diuretics and vasoconstrictor should be averted as they not merely could not stop capillary leakage but also lead to wound ischemia. Large amount of fluid infusion during burn shock exacerbates occurrence and progress of pulmonary edema. As a result, fluid volume should be controlled at the lowest effective level of circulating blood volume. Too much restriction of fluid volume, however, is not serviceable in patient's living through shock period, only to aggravate hypoxia, pulmonary perfusion decrease and to promote the occurrence and development of pulmonary edema. The primary principle for recovery is to tide over shock period monitored under hemodynamic monitoring. Swan-Ganz catheter monitoring is the alternative approach to maintain the level of pulmonary capillary wedge pressure (PAWP) at 6-12 mmHg. ${ }^{[12]}$

\subsection{Dr. Zai-qing Huang}

Dr. Zai-qing Huang is an anesthesiology physician at The Third Affiliated Hospital of Inner Mongolia Medical University, specializing in cardiac surgery anesthesia.

Occurrence of cardiac arrest after burns, mainly related to the following factors: (1) The incidence of hydrothermal flame burns is relatively high as flame burns are mostly associated with the inhalation burns. Though nudity wound is not much seen for patients with electrical injury, there are a lot of big deep devitalized tissues. Moreover, patients tend to have complication, such as complex brain injury, myocardial injury, are prone to respiratory and cardiac arrest so that close monitoring is essential for patients with electrical injury. (2) Burn area. Patients with large burn area are more prone to respiratory burns, cardiac arrest, due to systemic reactions intense, which may lead to edema at throat and tracheal mucosa and further result in respiratory obstruction, especially during the shock stage. ${ }^{[13]}$ Large area burn oozes more consumption and requires a large amount of fluid. Therefore, unreasonable fluid infusion, such as fluid is not in due time, composition is unreasonable and the amount of fluid is not enough, may aggravate ischemia and hypoxia. $^{[14]}$ (3) Burn sites. Compared with other burn sites, head and neck burns are more likely to inhalation burns, local edema, especially the throat, trachea mucosal edema, which may further bring about respiratory obstruction followed by respiratory and cardiac arrest. (4) Anesthesia accidents. Though standardized anesthesia medication being given, the patient was in critical condition with poor tolerance, hence some drugs have an inhibitory effect on respiratory.

Rescue measures: (1) Most commonly used measures. immediately solve the problem of airway obstruction, parallel external cardiac compressions are then performed, rescue agents (triple needle, respiratory stimulants, etc.) are administered to achieve treatment purposes from intravenous access. When chest compressions are proved to be invalid, thoracotomy surgery is suggested to conduct for heart squeezing. (2) Endotracheal intubation or incision. There are some shortcomings of intubation, such as easy suctioning, long term duration may cause larynx, vocal cords edema, ulceration, bleeding, etc. So many advocates of emergency tracheotomy, and keeps the intubation for a week. (3) Intracardiac injection or intravenous triple needle. We believe that there is a certain blindness in intracardiac injection, for example, it not only damages the myocardium (drug leakage, acupuncture damage), but also hurts the blood vessels, especially the coronary arteries. It may further give rise to myocardial hemorrhage, necrosis and acute myocardial tamponade. (4) Rescue agents. Patients with acute respiratory failure, which required inotropic agents, should use dobutamine. However dopamine should not be used since the drug increases cardiac output and boosts the rise of pulmonary artery wedge pressure 
(PCWP) which may lead to pulmonary edema, further aggravate respiratory failure. Naloxone, a respiratory stimulant, can improve anesthesia-induced respiratory depression. (5) Head cooling. It should be carried out simultaneously in breathing and cardiac arrest rescue.

\subsection{Dr. Li-hua Zhang}

Dr. Li-hua Zhang is the director of pharmacy at The Third Affiliated Hospital of Inner Mongolia Medical University, specializing in clinical pharmacy.

Systemic infection is the main causes of death in patients with extensive burns. As for the treatment of bacterial infections, drug selection should refer to the experience of recent resistance surveillance results of bacteria. Cefoperazone sulbactam is referred on the basis of clinical experience and monitoring results in burn units at our hospital. Amikacin injection (amikacin) $0.2 \mathrm{~g}$, ivgt, bid, was added according to the results of bacterial culture and sensitivity, wound dressing and intravenous nutritional support were strengthened on the first six days after the injury. On the first nine days after the injury, the patient's temperature up to $40^{\circ} \mathrm{C}$, heart rate 140-150 beats/min, with occasional delirium. Dissolved wound scab appeared with more secretions. Deep burns and a large area of tissue damage and necrosis provide a good place to bacterial growth, which poses a great threat to patients. The crust resection of residual wound, micro skin grafting, large allograft cover surgery is effective measure to control infection. Imipenem cilastatin sodium (imipenem) $1.0 \mathrm{~g}$, ivgt, tid; and vancomycin $0.8 \mathrm{~g}$, ivgt, bid, were injected since the patient was presented with restlessness and delirium on the first 10 days after injury. The patient's temperature was $38.9^{\circ} \mathrm{C}$, WBC $13.7 \times 10^{9} / \mathrm{L}$ after drug administration for 11 days so that imipenem and cilastatin sodium for injection and vancomycin were changed into piperacillin. Patients with wounds, sputum cultivated pseudomonas aeruginosa, acinetobacter baumannii, methicillinresistant Staphylococcus epidermidis, which turned out to be a variety of mixed bacterial infections. Imipenem and cilastatin sodium for injection and vancomycin for antiinfection were employed to cover Gram-positive bacteria, Gram-negative bacteria. The effect of clinical anti-infection treatment, however, is not ideal after drug administration for 11 days.

Currently, alternative antimicrobial agents for acinetobacter baumannii infections are limited, carbapenems, sulbactam is still the primary choice. As carbapenem antibiotic-resistant acinetobacter baumannii is increasing, pan-drug resistant acinetobacter baumannii (PDR-AB) brought great difficulties to clinical anti-infective therapy. A combined therapy, including carbapenems, amikacin or carbapenems and sulbactam were suggested for use for multi-drug resistant and pan-drug resistant strains. ${ }^{[15]}$ Pseudomonas aeruginosa isolation still ranked first due to the combined anti-infection effect of imipenem and cilastatin sodium, aminoglycoside. Therefore, a serious infection of Pseudomonas aeruginosa could be treated with the drugs above. It could be included from the discussion above that the anti-infection treatment of imipenem and cilastatin sodium alone is not ideal other than amikacin. Therefore, the clinical feasibility of using carbon carbapenems and amikacin in combination with/or sulbactam is referred to cure acinetobacter baumannii infection in patients with severe burns. Carbapenem antibiotic belongs to a time-dependent, a repeated intermittent dosing and a single continuous intravenous administration. As it has a longer post-antibiotic effect, it may be more appropriate to extend the dosing interval, 2-3 times a day to meet the needs of moderate to severe infections. Continuous intravenous administration has advantages over multiple doses for refractory pathogens, such as acinetobacter baumannii infections. ${ }^{[16]}$ In view of this, clinical dose three times a day will be changed to continuous intravenous treatment for acinetobacter baumannii infection in patients with severe burns.

Methicillin-resistant staphylococcus aureus and staphylococcus epidermidis (MRSA, MRSE) infections, vancomycin is preferred. Linezolid is the alternative choice when the expected effect was not achieved. The treatment of amikacin and vancomycin (or Norvancomycin) at the same time increased renal toxicity or neurotoxicity so that attention should be paid to clinical practice.

The patients with systemic flame burns 95\%, 90\% thirddegree burns, deep second degree burns 5\%, for large area deep burn, was discharged from the hospital after treatment for 108 days. It is analyzed that the successful treatment of the patients is due to timely resection of necrotic tissue and wound closure, application of nutritional support, reasonable choice of antimicrobial agents as well as effective anti-shock therapy.

\subsection{Dr. Ling-feng Wang}

Dr. Ling-feng Wang is the chief physician and professor at The Third Affiliated Hospital of Inner Mongolia Medical University and Inner Mongolia Institute of burns, specializing in burn wound healing and tissue engineering, mechanism of bacterial resistance.

MODS remains a difficult issue for the doctors to establish the diagnosis and appropriate treatment for the patients with severe burns accompanied by deeper wound to thirddegree, fourth-degree, complex disease and system organ problems. ${ }^{[17]}$

The features of the disease. (1) Patients with burn area up to $95 \%$, the depth of deep burns to third-degree mainly for $90 \%$ provides pathological basis for the occurrence of MODS. (2) The patient was presented with organ dysfunction and emergency gastrointestinal bleeding at the earlier 
stage during the shock stage and long-distance transport. (3) Simultaneous or sequential clinical manifestation of two or more sequential organ function disorder in a critical condition without effective treatment. (4) Infection is an important factor in inducing and aggravating MODS. Excessive inflammation caused by infection aggravates the condition and initiates a "second strike", so that inflammatory cells in the "excited state" are more likely to release excessive cellular and humoral media, which enlarged inflammation, resulting in further tissue damage and organ dysfunction. (5) MODS may be cured with effective treatment so that the performance of organ dysfunction is reversible. Organ function is expected to recover and the patient to be cured when the pathogenesis blocked by timely treatment.

Diagnosis. The diagnosis of MODS is not difficult in accordance with the revised edition in 1995 about the staging diagnosis and level of severity of the disease. Early and accurate detection of systemic inflammatory response syndrome (SIRS) and organ dysfunction serves as the key to early diagnosis of MODS. ${ }^{[18]}$ The primary clinical manifestation for MODS evolution is sequential changes, usually starting from a multi-organ, and then the other organ lesions. A complete diagnosis of MODS should include predisposing factors, SIRS and MODS three parts. ${ }^{[19]}$ The clinical manifestation of the patients are: (1) Severe burns, infections and a lot of necrotic tissue, such as MODS retention incentive; (2) SIRS; (3) Two or more systems or organ dysfunction (gastrointestinal tract, blood clotting function, lung, brain, heart, liver dysfunction). The diagnosis of MODS is confirmed.

Differential diagnosis. MODS is a kind of serious complications for severely burned patients during the treatment. SIRS appear along with sequential multi-organ dysfunction. There is a process of development of organ dysfunction, including dysfunction period, exhaustion and failure of early stage and other different clinical manifestations according to revised edition in 1995 about staging diagnosis and level of severity of MODS via Lushan National Critical Care Medicine Conference. MODS is an accumulating result of multiple organ dysfunction so that dynamical observation of each affected organ damage and its development is required. Besides that, determining the severity of organ dysfunction and timely detecting of abnormal organ function are essential. Accurate differential diagnosis should base on comprehensive issues related to each organ dysfunction. (1) Diagnosis is a judgment on the basis of all the integration of information, is a kind of comprehensive analysis of a variety of symptoms, signs and relevant inspection data, is a process of identifying various similar disease. MODS is a serious disease for patients with severe burns. A comprehensive analysis of the symptoms, signs and checking data is suggested to conduct, detection of organ dysfunction occurrence and structural and functional abnormalities, making accurate determination are required. (2) During the courses of treatment for severe burns, the patients are commonly ac- companied by SIRS. MODS often occurs on heavily damaged organs from an early start, gradually sequential lead to other organ damage, failure as the disease progresses. The first damaged organ varies in different cases, consequently, the occurrence of secondary MODS sequential involvement of organs is different as well. Hence, the diagnosis requires a concrete condition. (3) The severity of affected organ dysfunction varies with changes in the disease of MODS so that impaired period, failure of early stage and failure period could be converted to each other. Prompt finding of typical performance of involved organs is of an assist to make timely diagnosis. In addition to that, some atypical clinical manifestations should paid attention to avoid misdiagnosis.

Treatment schedule. Pathogenesis combined with prevention and treatment of organ function plays a critical role in the treatment of MODS among patients with large burn. But beyond that, infection control, wounds resection and being away from a "second strike" could not be ignored. In other words, to solve the problem of burn wounds remains imperative for possible recovery of damaged organ or failure organs. Currently, an effective method of containing the occurrence of MODS and its pathophysiological process is far from satisfactory so that the treatment of MODS mainly relies on organ function support.

(1) Fast, efficient burn shock resuscitation. The "first strike" for patients suffered from severe burns is the period of low perfusion burn shock, tissue ischemia and hypoxia. Timely and effective rehydration therapy to prevent and correct the dominant or recessive shock, ensure the organization blood, oxygen, is widely accepted. Liquids burn shock therapy as the center of a comprehensive treatment is applied upon a deeper understanding of pathophysiological changes of burns, so that most patients can tide over the shock stage smoothly. Since then, there is a significant decrease of severe cases of visceral dysfunction during shock stage. Fast and efficient burn shock resuscitation of burn patients is not only the basic treatment but also is the key to prevention and treatment of early MODS. The purpose of fluid therapy is to maintain the effective volume. No matter what burn shock rehydration formula was employed, the calculated amount is an estimate of fluid. Therefore, personalized treatment is on the basis of specific clinical circumstances. Intravenous access and rapid infusion were performed upon his admission to restore effective circulating volume of blood and shorten the tissue hypoperfusion time. More rapid infusion should be within 1-2 hours to prevent shock as soon as possible and further damage to internal organs, especially for patients with delayed recovery. Rapid fluid infusion is not the same as large amount of fluid, which should be operated under shock monitoring to avoid the burden on cardiology and pulmonary, and alternate input of crystals, colloids and water should be focused. In addition to burn shock resuscitation fluid therapy, the use of vitamin $\mathrm{C}$, vitamin $\mathrm{E}$, glutamic acid, $\beta$-carotene and diluted mannitol could be applied at the same time to mitigate the damage of oxygen free radicals. 
Anisodamine, salvia and other drugs were effective in the visceral microcirculation improvement and early protection visceral functions.

(2) Early surgical removal of necrotic tissue for prevention of infection. Necrotic tissue of burn wound is the basic cause of pathophysiological changes and excessive systemic inflammatory. Necrotic tissue and wound infections continuously release toxic substances, activate many inflammatory mediators involved in MODS occurrence and progress before complete healing of burn wounds. The burn wound infection rate is higher and trigger a major source of systemic infection during the treatment of burns. Therefore, early surgical removal of necrotic tissue in burn wounds, especially necrosis in deep burn wound, autologous skin or a variety of mixed autologous skin graft to cover the wound substitutes, early burn wound repair and burns pathophysiological changes control are the fundamental measures of MODS treatment. Surgical removal of scab carries best outcome for patients with large deep burn area to reduce the number of pathophysiology of necrotic tissue caused by the release of toxic substances, if the condition is allowed. In addition, it would be better if the removal scab is up to about $40 \%$ total body surface area (TBSA). The treatment of infected burn wound is particularly important when the burn wound infection has become a major factor that contributes to the incidence and progress of MODS. In that case, we must resolutely and decisively provide adequate drainage of wound infection, remove necrotic tissue as early as possible, and cover the wound in a timely manner. ${ }^{[20]}$

(3) Early application of enteral nutrition for prevention of intestinal bacteria and endotoxin translocation. ${ }^{[21]}$ Recent studies further indicate that the gut, as the body's largest "bacteria reservoir" and "endotoxin reservoir", is involved in in the pathophysiology of SIRS and MODS process for its unique physiological environment in the body. Intestinal barrier is damaged, intestinal immune function is suppressed, intestinal bacterial translocation and endotoxin, bacteria, antibodies media continue to enter the blood and lymph, resulting in a variety of inflammatory mediator release, trigger and aggravate uncontrolled inflammatory response syndrome and the occurrence of SIRS intestinal damage, which creates a vicious cycle and eventually leads to MODS. The early application of enteral nutrition support has become an important means of protecting the intestinal mucosa, preventing bacterial translocation, lowering stress metabolic reactions and reducing gastrointestinal complications since gastrointestinal barrier mechanisms and intestinal infection was proposed in recent years. Studies have shown that early enteral nutrition, especially with immunomodulatory components such as arginine, polyunsaturated fatty acids, ribonucleic acid, glutamine and other nutrients are effective to prevent intestinal mucosal atrophy, reduce intestinal bacterial translocation and septic concurrent disease incidence. Enteral nutrition not only provides appropriate nutrients for the body but also directly supplies special nutrients for the gut itself. On the other hand, enteral nutrition can promote the secretion of pancreatic and gastrointestinal hormones through the stimulation of the gastrointestinal mucosa, and maintain normal intestinal mucosa structure and barrier function. Currently, the market alternative enteral nutrition products are more available for use, so the selection is depended on specific clinical situation.

(4) Strengthen internal organs protection and safeguard the overall organ function. The main causes of severe burn death are due to MODS. The possibility of the occurrence of MODS is increasing along with the increasing of burn areas of third-degree, fourth-degree. All the internal organs burn patients are with pathophysiological changes of different degrees from early burn "first strike" of burn to "second hit" of the treatment process. Therefore, the protection of internal organs seems very important during the treatment courses. Moreover, always pay attention to the maintenance of patients organ function, prevent the occurrence of visceral organ dysfunction and injury. For example, fast early resuscitation to restore blood supply to tissue cells, application of oxygen free radicals antagonists were employed and reduce the damage of oxygen free radicals. In addition to that, early application of drugs including anisodamine and salvia was put into use to improve blood circulation visceral, along with early parenteral nutrition.

(5) Comprehensive treatment of injured and failure organs. Targeted and effective measures should be performed to treat impaired function of the failure organ upon the occurrence of MODS.

Improve heart function and blood circulation. Appropriate treatment should be taken for cardiac arrhythmia, and even cardiac arrest, as well as peripheral circulatory disorders after the occurrence of MODS. Closely monitor cardiac function, preload, afterload and effective circulating volume. Determining the infusion volume, infusion rate, reasonable allocation of crystalloid solution and colloid solution, reasonable application of vasoactive drugs according to the condition of the disease. The factors affecting heart function and blood circulation should be taken into account in the course of treatment, while systematic treatment should not be ignored.

Strengthen respiratory support. The incidence of lung function impairment is high with the most obvious symptoms and requires a longer use of mechanical ventilation for burn patients with MODS. Pulmonary is usually regarded as the most involved organ by MODS, therefore the treatment of pulmonary function seems crucial and calls for early ventilator-assisted therapy. Its application could not be postponed until respiratory failure. Furthermore, strengthening airway humidification and bronchoalveolar lavage is critical measure to clear respiratory secretions, prevent pulmonary infection, and protect bronchial cilia movement.

Maintenance of renal function. The incidence rate of im- 
paired renal function and renal failure are higher due to delayed resuscitation, unsmooth condition during shock stage and serious infection. ${ }^{[22]}$ The basic measures are to maintain effective blood volume, avoid or reduce shrinkage with a vascular medicine, ensure and improve renal perfusion. When renal impairment or failure is detected, bedside blood purification, dialysis and other measures to block further damage kidney function is required at once. Kidney function can get a better recovery after aggressive treatment. In addition, CRRT is an indispensable means to effectively reduce serum inflammatory mediators in patients with burns and cytokines, and to prevent SIRS, MODS.

Treatment of the gastrointestinal tract and liver function. The gastrointestinal tract then receives more attention after the incidence and progress of MODS due to indepth study of MODS. Early intestinal barrier function was impaired 5 minutes after burn, and the impairment peaked within 4 hours. Intestinal bacterial translocation and enterogenic infection as the "second strike" contributes to the occurrence and progress of MODS. In addition to traditional treatment of gastrointestinal hemorrhage by relieving hyperacidity and stopping bleeding, the application of somatostatin enables gastrointestinal hemorrhage to be better controlled. Meanwhile, pay attention to liver function protection, reduce the burden on the liver and prompt the recovery of damaged functions.

Prevention of clotting dysfunction. Causes of abnormal clotting function is mainly due to excessive consumption of clotting substances, infections are usually regarded as the most sudden factor. Early examination, early detection and early intervention are efficient to prevent serious consequences caused by clotting dysfunction, such as DIC. ${ }^{[23]}$

Brain dysfunction. Brain dysfunction serves as an indicator of severe condition, non-effective treatment of MODS as well as the continuous progress of MODS. The ease of brain function abnormality is ensured along with the condition of MODS control. Protection of cerebral tissue is the main measure of the treatment.

Treatment of metabolic and internal environment disorders. Metabolic and internal environment disorders induced by MODS are secondary in nature, which should be treated at once and the comprehensive treatment of burns treatment and MODS strengthened at the same time. It could be cured along with burn wound repair, restoration of damaged organ function.

In addition to its primary burn wounds change, MODS patients often carries some common clinical features according to comprehensive analysis of MODS after burn cases. That is, thorough knowledge of the common clinical features is the key to prevent missed diagnosis or misdiagnosis of MODS in advance. The expectation of reducing its incidence and raising the cure rate could be achieved by early detection, intervention and treatment combined with MODS prevention and control. Patients with MODS after a typical burn often experienced longer hypovolemic shock and different degrees of reperfusion injury caused by blood volume rehabilitation, were often presented with incomplete or delayed recovery. Patients, who have lived through the first hit, experienced a relatively stable period when haemodynamics index is relatively stable. The potential risk of gastrointestinal ischemia, metabolic disorders and mild, moderate of inflammatory reaction could not be ignored. The patient underwent a second hit (such as burn wound sepsis, intestinal infection, etc.) after a few day or weeks, was presented with high metabolism, high power cycle as the main clinical manifestation of SIRS or sepsis symptoms, which eventually result in an organ dysfunction or even failure, the further sequential development of other organ dysfunction or even failure. On one hand, early detection, diagnosis and treatment of organ dysfunction during this period remains imperative for possible recovery. On the other hand, attach importance to the atypical symptoms which is a useful tool to aid in the diagnosis of MODS. For example, we have to identify the causes of oliguria, anuria or polyuria, for patients treated with delayed resuscitation during shock period, it being the capacity problem or impaired renal function, to take the corresponding treatment measures. An estimate of degrees of lung function impairment is essential for serious burn patients with inhalation injury to determine whether tracheotomy or ventilator support is needed. Poor judgment of impaired lung function often brings about rapid deterioration of MODS disease. The causes of gastrointestinal bleeding is due to overweight emergency response, primary disease of digestive tract, gastrointestinal stress ulceration and bleeding, gastrointestinal function failure or coagulation function failure caused by hemorrhage of digestive tract, and its diagnosis mainly relies on a comprehensive analysis.

The main clinical manifestations of SIRS are secondary to a variety of blows sustained high systemic metabolism, hyperdynamic circulation and excessive inflammation. In addition to clinical manifestations of body temperature, respiratory frequency, heart rate and white blood cell count, classification ratio change, uncontrolled release of a variety of cytokines and inflammatory mediators also serves as an indicator, whose extraordinary intense inflammatory response will damage the normal tissue and organs. ${ }^{[24]}$ The clinical manifestation of SIRS is the most frequent symptoms for patients with severe burn and indicates the incidence of MODS, therefore, clinical attention should be paid to the treatment and monitoring of SIRS, close observation of the disease, early detection of the symptoms, and timely treatment is required.

The system or organ dysfunction often shows a relatively regular order during the MODS development process. Lung is mostly observed as the first organ failure with high rate in patients with serious burns at clinical practice due to its anatomical features of the vulnerable against a variety 
of pathogenic factors, convenient observation, monitoring and other factors. Besides that, lung not only functions for gas exchange but also serves as a place for some hormones and medium production or inactivation. As a consequence, pulmonary dysfunction not only leads to oxygen delivery reduction to systematic organs, tissue oxygen metabolism disorders but also result in content changes of some medium circulating in the blood, such as kallikrein, 5-hydroxytryptamine and angiotensin. In recent years, the role of the gastrointestinal tract in the form of MODS has been paid an increasing attention. Many research reports demonstrated the earlier damage or failure of intestinal mucosal barrier function in the pathogenesis of MODS, which is especially prominent in severe burn patients complicated with shock, reperfusion injury. The gastrointestinal tract is the body's largest reservoir of bacteria and endotoxin so that intestinal barrier damage can cause intestinal bacterial translocation and endotoxemia in liver, leading to activation of monocyte/macrophage system, initiating the systemic inflammatory response. For all these reasons, monitoring and protection of the lung and intestinal barrier function is of special significance for the early diagnosis and treatment of MODS. Liver function damage and failure, gastrointestinal bleeding and blood system disorders, heart or renal failure is usually MODS terminal performance along with the development of MODS disease. ${ }^{[25]}$

\section{References}

[1] Field JM, Hazinski MF, Sayre MR, et al. American Heart Association Guidelines for Cardiopulmonary Resuscitation and Emergency Cardiovascular Care. Circulation. 2010; 122(18 Suppl 3): 640-656. PMid:20956217. http://dx.doi.org/10.1161/ CIRCULATIONAHA.110.970889

[2] Kitamura T, Iwami T, Kawamura $\mathrm{T}$, et al. Conventional and chest-compression-only cardiopulmonary resuscitation by bystanders for children who have out-of-hospital cardiac arrests: A prospective, nationwide, population-based cohort study. Lancet. 2010; 375(9723): 1347-1354. http://dx.doi.org/10.1016/ S0140-6736(10)60064-5

[3] Callaway CW, Schmicker R, Kampmeyer M, et al. Receiving hospital characteristics associated with survival after out-of-hospital cardiac arrest. Resuscitation. 2010; 81(5): 524-529. PMid:20071070. http://dx.doi.org/10.1016/j.resuscitation.2009.12. 006

[4] Zongcheng Yang. The treatment of Burns. 3rd ed. BJ: The People's Medical Publishing House. 2006; 273-276.

[5] Zhang Zheng, Liang Li. Severe burn complicated with acute respiratory distress syndrome - related factors and diagnosis and treatment. Chinese Journal of practical medicine. 2009; 4(24): 61-63.

[6] Linsen Fang, Delin Hu, Youxin Yu, et al. Protective mechanical ventilation in a large area of Burn Complicated with application in the treatment of inhalation injury. Acta Universitatis Medicinalis Anhui. 2011; 6(9): 943-946.

[7] Jieming He, Qinglin Hao. Research Progress of cytokines and acute lung injury. Medical Review. 2009; 15(3): 385-389.

[8] Jun Liu. Clinical application on continuous renal replacement therapy in severe Burn Patients. Journal of Traumatic Surgery. 2008; 10(5): 466.

[9] Qun Jiang, Weifeng Tu. Sufentanil Pretreatment on protective effect of acute gastric mucosal lesions in rats and its relation with acid sensing ion channels. Journal of Southern Medical University. 2010; 30(5): 1099-1102.

[10] Dawei Liu. Practical critical care medicine. BJ: People's Medical Publishing House. 2011; 423-430.

[11] Xiang Zhang. ICU pharmacotherapeutice. BJ: Chemical Industry Press. 2010; 46-55.

[12] Chunting Wang. Avoid common errors in ICU. BJ: People's Medical Publishing House. 2008; 719-721.

[13] Zongcheng Yang. Improve the way of early rehydration reduce visceral damage of early postburn. Chinese Journal of Burns. 2005; 21(3): 163-164.

[14] Wei Lu, XiaZhao Fan, HuanJing Ning, et al. Five burned patients with success or failure cardiac rescue cause analysis. Chinese Journal of Burns. 2005; 21(2): 160.
[15] Fangmei Zhou, Fei Xiaxue, Qing Ye, et al. study on Pan - resistant acinetobacter infection of plasmid to mediated carbon penicillium of butadiene. Zhejiang Clinical Medical Journal. 2012; 14(5): 13-515.

[16] Can Chen, Ruiqin Zhang, Jingju Duan, et al. Survey on rational use of drugs about carbon penicillium vinyl in our hospital. Chinese Remedies and Clinics. 2012; 12(1): 57-58

[17] Nguyen LN, Nguyen TG. Characteristics and outcomes of multiple organ dysfunction syndrome among severe-burn patients. Burns. 2009; 35(7): 937-941. PMid:19553020. http://dx.doi.org/ 10.1016/j.burns.2009.04.015

[18] Ferreira AM, Sakr Y. Organ dysfunction: general approach, epidemiology, and organ failure scores. Semin Respir Crit Care Med. 2011; 32(5): 543-551. PMid:21989690. http://dx.doi.org/ $10.1055 / \mathrm{s}-0031-1287862$

[19] Klein Klouwenberg PM, Ong DS, Bonten MJ, et al. Classification of sepsis, severe sepsis and septic shock: The impact of minor variations in data capture and definition of SIRS criteria. Intensive Care Med. 2012; 38(5): 811-819. PMid:22476449. http: //dx.doi.org/10.1007/s00134-012-2549-5

[20] Huang LF, Yao YM, Li JF, et al. Reduction of plasma gelsolin levels correlates with development of multiple organ dysfunction syndrome and fatal outcome in burn patients. PLoS One. 2011; 6(11): e25748. PMid:22069445. http://dx.doi.org/10.1371/ journal. pone. 0025748

[21] Mosier MJ, Pham TN, Klein MB, et al. Early enteral nutrition in burns: Compliance with guidelines and associated outcomes in a multicenter study. J Burn Care Res. 2011; 32(1): 104-109. PMid:21116188. http://dx.doi.org/10.1097/BCR. ob013e318204b3be

[22] Steinvall I, Bak Z, Sjoberg F. Acute kidney injury is common, parallels organ dysfunction or failure, and carries appreciable mortality in patients with major burns: A prospective exploratory cohort study. Crit Care. 2008; 12(5): 124. PMid:18847465. http: //dx.doi.org/10.1186/cc7032

[23] Lippi G, Ippolito L, Cervellin G. Disseminated intravascular coagulation in burn injury. Semin Thromb Hemost. 2010; 36(4): 429-436. PMid:20614394. http://dx.doi.org/10. $1055 / \mathrm{s}-0030-1254051$

[24] Mace JE, Park MS, Mora AG, et al. Differential expression of the immunoin flammatory response in trauma patients: burn $v s$. non-burn. Burns. 2012; 38(4): 599-606. PMid:22103986. http: //dx.doi.org/10.1016/j.burns. 2011.10.013

[25] Guttormsen AB, Onarheim H, Thorsen J, et al. Treatment of serious burns. Tidsskr Nor Laegeforen. 2010; 130(12): 1236-1241. PMid:20567275. http://dx.doi.org/10.4045/tidsskr.08. 0391 